TRANSACTIONS OF THE

AMERICAN MATHEMATICAL SOCIETY

Volume 355, Number 3, Pages 1167-1182

S 0002-9947(02)03095-7

Article electronically published on October 30, 2002

\title{
SPHERICAL MAXIMAL OPERATOR ON SYMMETRIC SPACES OF CONSTANT CURVATURE
}

\author{
AMOS NEVO AND P. K. RATNAKUMAR
}

\begin{abstract}
We prove an endpoint weak-type maximal inequality for the spherical maximal operator applied to radial funcions on symmetric spaces of constant curvature and dimension $n \geq 2$. More explicitly, in the Lorentz space associated with the natural isometry-invariant measure, we show that, for every radial function $f$,

$$
\|\mathcal{M} f\|_{n^{\prime}, \infty} \leq C_{n}\|f\|_{n^{\prime}, 1}, \quad n^{\prime}=\frac{n}{n-1} .
$$

The proof uses only geometric arguments and volume estimates, and applies uniformly in every dimension.
\end{abstract}

\section{InTRODUCTION}

Let $\sigma_{t}$ denote the normalised surface measure on the sphere $|x|=t$ in $\mathbb{R}^{n}$. The celebrated spherical maximal theorems of E. Stein ([S1]) (for $n \geq 3$ ) and J. Bourgain ([B] $)$ (for $n=2$ ) assert that the spherical maximal operator $\mathcal{M} f(x)=$ $\sup _{t>0}\left|f * \sigma_{t}(x)\right|$ satisfies the strong $L^{p}$-maximal inequality on $L^{p}\left(\mathbb{R}^{n}\right)$, namely,

$$
\|\mathcal{M} f\|_{p} \leq C_{p}(n)\|f\|_{p},
$$

whenever $f \in L^{p}\left(\mathbb{R}^{n}\right), p>n /(n-1), n \geq 2$.

Similarly, consider the maximal operator associated with averaging on geodesic spheres in hyperbolic $n$-space $H^{n}$. This operator satisfies the same strong maximal inequalities as its Euclidean counterpart. The first proof of this result for $n \geq 3$ was given by El Kohen ([K]; see [N2] and [NS] for an alternative proof), and very recently the case $n=2$ was settled by Ionescu [I]

The proofs of all the foregoing results rely on intricate spectral considerations and detailed information regarding harmonic analysis on the underlying spaces. They are optimal in the sense that for every $p \leq \frac{n}{n-1}$, there exists a function $f$ in $L^{p}(X)$ for which the spherical maximal operator satisfies $\mathcal{M} f(x)=\infty$ for almost every $x \in X$ (see [SWe], [N2] and [I] ).

A natural generalization of the foregoing set-up is obtained by considering the geodesic sphere $S_{t}$ (with its Riemannian measure) in symmetric spaces of compact and non-compact type, and the associated averaging and maximal operators. An even more challenging goal is that of establishing analogous maximal theorems for

Received by the editors June 5, 2000.

2000 Mathematics Subject Classification. Primary 43A85; Secondary 43A18.

Key words and phrases. Symmetric spaces, constant curvature, spherical means, maximal function.

The first author was supported by Technion V.P.R. fund-E. and J. Bishop research fund, and the second author was supported by the fund for the promotion of research at the Technion. 
singular spherical averages on arbitrary Gelfand pairs. These include such important examples as sphere averages on the Heisenberg group (see [C], [NT]), and, more generally, spherical averages associated with Cartan motion groups.

However, the spectral theory available for general Gelfand pairs, and even for higher-rank symmetric spaces, is far from sufficient to derive results analogous to the one above. It therefore seems desirable, and necessary, to develop more direct geometric methods to make some progress in this direction.

In the present paper, we will consider the basic case of symmetric spaces of constant curvature. We establish an end-point weak-type maximal inequality for the spherical maximal operator (at the point $\frac{n}{n-1}$ ) acting on radial functions. The argument is based solely on geometric considerations and volume estimates. It does not use spectral theory, and applies equally well to Euclidean spaces, hyperbolic spaces and spheres of arbitrary dimension $n \geq 2$.

To formulate the result precisely, let $X$ be a symmetric space of constant curvature and dimension $n \geq 2$. Let $\mathcal{A}_{t} f(x)$ denote the average of a function $f \in C(X)$ over the geodesic sphere of radius $t$, centered at $x$. Consider the maximal operator (well-defined for $f \in C(X)$ )

$$
\mathcal{M} f(x)=\sup _{t>0}\left|\mathcal{A}_{t} f(x)\right| .
$$

We let $\|\cdot\|_{p, q}$ denote the norm in the Lorentz space $L^{p, q}\left(X, \mu_{n}\right)$, where $\mu_{n}$ is the measure on $X$ invariant under the isometry group of $X$ (see $\S 3$ for more on Lorentz spaces). Finally, let $n^{\prime}=n /(n-1)$ be the index conjugate to $n$. We can now formulate our main result:

Theorem 1.1. Let $X$ be a symmetric space of constant curvature, $\operatorname{dim} X=n \geq 2$. Then the maximal operator $\mathcal{M}$ satisfies the inequality

$$
\|\mathcal{M} f\|_{n^{\prime}, \infty ; \mu_{n}} \leq C_{n}\|f\|_{n^{\prime}, 1 ; \mu_{n}}
$$

for some constant $C_{n}$ independent of $f$, whenever $f$ is a radial function on $X$.

In view of the obvious estimate

$$
\|\mathcal{M} f\|_{\infty} \leq\|f\|_{\infty}
$$

Theorem 1.1 yields, by interpolation, a proof of the norm boundedness of the spherical maximal operator acting on radial functions in $L^{p}(X), p>\frac{n}{n-1}$, when $X$ is a symmetric space of constant curvature.

We note that in the Euclidean case the end-point result was already established by J. Bourgain B2, for $n \geq 3$ (and arbitrary functions), using spectral considerations. For radial functions on $\mathbb{R}^{n}$ it was established for all $n \geq 2$ by M. Leckband $\mathrm{L}$. Another proof of the latter result is due to $[\mathrm{KR}]$, and served as the starting point of the discussion that follows. In the non-Euclidean case, namely for spherical and hyperbolic geometry, the end-point result above is new.

\section{Symmetric spaces of Constant CuRvature}

Let $X=X(\kappa, n)$ be a complete, simply connected $n$-dimensional Riemannian manifold of constant curvature $\kappa \in\{0,1,-1\}$. Then $X$ is uniquely determined up to isometric equivalence [Hi]. When $\kappa=0$, one obtains $X=\mathbb{R}^{n}$ with the Euclidean metric $d s^{2}=d x_{1}^{2}+\cdots+d x_{n}^{2}$. For $\kappa \neq 0$, these spaces can be visualised as the $n$ dimensional quadratic surfaces in $\mathbb{R}^{n+1}$ given by the equation $Q_{\kappa}(x, x)=1$, where $Q_{\kappa}(x, x)=\kappa\left(x_{1}^{2}+\cdots+x_{n}^{2}\right)+x_{n+1}^{2}$. Thus when $\kappa=1$, one obtains the unit sphere 
$S_{1}^{n}$ in $\mathbb{R}^{n+1}$. For $\kappa=-1$, one obtains the $n$-dimensional hyperboloid of two sheets in $\mathbb{R}^{n+1}$. We will use the notation $H^{n}$ for the $n$-dimensional hyperbolic space, which is identified with one of the sheets.

The metric $d(x, y)$ on $X(\kappa, n)$ when $\kappa \neq 0$ is given by the implicit equation $\psi(d(x, y))=Q_{\kappa}(x, y)$, where $\psi$ is a function determined by the curvature. Explicitly, $\psi(t)=\cos t, 0 \leq t \leq \pi$, for the sphere, and $\psi(t)=\cosh t$ for hyperbolic space. We also define $\psi(t)=t^{2} / 2$ in the Euclidean case. Given three points $x_{1}, x_{2}, x_{3}$ in $X(\kappa, n), \kappa \neq 0$, we view them as vectors in the corresponding linear space (defined above). Consider the determinant of the Gram matrix associated with $Q_{\kappa}$ :

$$
\delta\left(x_{1}, x_{2}, x_{3}\right)=\operatorname{det}\left(Q_{\kappa}\left(x_{i}, x_{j}\right)\right)=\operatorname{det} \psi\left(d\left(x_{i}, x_{j}\right)\right) .
$$

Clearly $\delta$ depends only on the mutual distances $d\left(x_{i}, x_{j}\right)$. Set $r=d\left(x_{1}, x_{2}\right), s=$ $d\left(x_{2}, x_{3}\right), t=d\left(x_{3}, x_{1}\right), p=\frac{1}{2}(t+r+s)$, and $\Delta(r, t, s)^{2}=\delta\left(x_{1}, x_{2}, x_{3}\right)$. Then a straightforward computation gives the following factorization (see, e.g., [Iv], pp. $71,87)$ :

$$
\Delta(r, t, s)^{2}=4 \varphi(p) \varphi(p-r) \varphi(p-t) \varphi(p-s) \geq 0 .
$$

Here $\varphi(t)$ is given by $\sin t, \sinh t$ and $t$, in spherical, hyperbolic and Euclidean geometries, respectively.

We note that in Euclidean space, the kernel $\Delta(r, t, s)$ is simply twice the area of a triangle with side lengths $r, t$ and $s$.

The simply connected spaces of constant curvature are symmetric spaces. In particular, these spaces can be realised as homogeneous spaces $G / K$, where $G=$ $\operatorname{Isom}^{0}(X)$ is the connected component of the group of isometries of $X$, and $K$ is the subgroup of $G$ that fixes a given point $p_{0} \in X$. As is well known, the pair $(G, K)$ is a Gelfand pair; namely, the convolution algebra of bi- $K$-invariant $L^{1}$-functions on $G$ is commutative. The pairs $(G, K)$ for constant curvature symmetric spaces are given as follows.

When the curvature is positive, the corresponding space is $S^{n}$. The group $S O(n+1)=O^{0}(n+1)$ of special orthogonal matrices acts isometrically and transitively on the $n$-dimensional sphere $S^{n} \subset \mathbb{R}^{n+1}$. The subgroup of $S O(n+1)$ that leaves the north pole $e_{1}=(0, \cdots, 1)$ invariant can be identified with $S O(n)$. The diffeomorphism $g \cdot S O(n) \rightarrow g \cdot e_{1}$ establishes the identification of $S^{n}$ with $S O(n+1) / S O(n)$.

When the curvature is zero, the corresponding space is Euclidean $n$-space. The isometry group is the Euclidean motion group $M(n)$, the group generated by translations and rotations in $\mathbb{R}^{n}$. Clearly $M^{0}(n)$ is the semidirect product of $\mathbb{R}^{n}$ and $S O(n)$, with the group law defined by $\left(x_{1}, k_{1}\right) \cdot\left(x_{2}, k_{2}\right)=\left(x_{1}+k_{1} x_{2}, k_{1} k_{2}\right)$. The subgroup of $M^{0}(n)$ that leaves the origin in $\mathbb{R}^{n}$ invariant is clearly the group $S O(n)$, and as before we see that $\mathbb{R}^{n}$ can be identified with the homogeneous space $M^{0}(n) / S O(n)$.

Finally, when the curvature is negative, the corresponding space is hyperbolic $n$-space. The isometry group is the Lorentz group $O(n, 1)$. It is the group of all $(n+1) \times(n+1)$ matrices that leaves the bilinear form

$$
Q(x, y)=-\left(x_{1} y_{1}+\cdots+x_{n} y_{n}\right)+x_{n+1} y_{n+1}
$$

invariant. In other words, it is the subgroup of $G L(n+1, \mathbb{R})$ given by

$$
O(n, 1)=\left\{g \in G L(n+1, \mathbb{R}): g^{t} J g=J\right\}
$$


where $J$ denotes the matrix $\operatorname{diag}\{-1, \cdots,-1,+1\}$. The group $S O^{0}(n, 1)$ acts transitively on hyperbolic space $H^{n}$. The subgroup of $S O^{0}(n, 1)$ that leaves invariant the point $(1, \cdots, 0,0)$ on $H^{n}$ in the hyperboloid model, can be identified with $S O(n)$. Again as in the previous cases, we can identify $H^{n}$ with the homogeneous space $S O^{0}(n, 1) / S O(n)$.

Notation. In this paper, $X$ will denote the symmetric space, $G=I_{\operatorname{som}}^{0}(X)$ the connected component of the group of isometries of $X$, and $K$ the stability group of a given point. $K$ is isomorphic to $S O(n)$ in all three geometries of dimension $n$. We call the point in the symmetric space $G / K$ corresponding to the coset $K$ the origin in $X$, and denote it by $o$.

The main feature of the structure theory of $G$ that is relevant to our discussion is the existence of a Cartan decomposition. Here such a decomposition takes the form $G=K A_{+} K$, where $K=S t_{G}(o)=S O(n)$ and $A$ is a one-dimensional Abelian subgroup of $G$. Parametrizing $A$ by $t \in \mathbb{R}$, we see that any element $a_{t} \in A$ has a matrix representation as follows.

In the Euclidean case,

$$
a(t)=\left(\begin{array}{ll}
I_{n} & t e_{1} \\
0 & 1
\end{array}\right),
$$

where $e_{1}=(1,0, \cdots, 0)^{t r} \in \mathbb{R}^{n}$.

For the sphere and the hyperbolic space, $a_{t}$ has the matrix representation

$$
a(t)=\left(\begin{array}{lll}
\psi(t) & 0 & \psi^{\prime}(t) \\
0 & I_{n-1} & 0 \\
\varphi(t) & 0 & \varphi^{\prime}(t)
\end{array}\right),
$$

where $I_{n-1}$ is the $(n-1) \times(n-1)$ identity matrix.

Since $X=G / K$ is a homogeneous space, we can consider functions on $X$ as right- $K$ invariant functions on $G$. Therefore, we can write the spherical mean value operator on $X$ as a convolution operator on $G$, given by

$$
\mathcal{A}_{t} f(g K)=f * \tilde{\sigma}_{t}(g)=\int_{G} f\left(g y^{-1}\right) d \tilde{\sigma}_{t}(y),
$$

where $\tilde{\sigma}_{t}$ is the unique bi- $K$-invariant probability measure on $G$ projecting to $\sigma_{t}$ under the canonical map $G \rightarrow G / K$ (note that $\tilde{\sigma}_{t}$ is a symmetric measure).

We note that when $f$ is radial we have $f * \tilde{\sigma}_{t}=\tilde{\sigma}_{t} * f$, so that in that case

$$
\mathcal{A}_{t} f(g K)=\int_{G} f\left(y^{-1} g\right) d \tilde{\sigma}_{t}(y)=\tilde{\sigma}_{t} * f(g) .
$$

The starting point of our analysis of spherical averages of radial functions is a formula which expresses the spherical average as a kernel operator. We will give a simple geometric proof of the formula for completeness. We refer to [FJ-K, $\$ 4$ ] or [K] $\S 7]$ for an analytic discussion of the kernel in the case of real rank-one groups. For the case of plane geometries, see, e.g., [H2, p. 369] or [W]. A simple group-theoretic proof for hyperbolic spaces can be found in [N1].

First, let $f$ be a radial function on $X$ or, equivalently, a bi- $K$-invariant function on $G$. Then in view of the $K A_{+} K$ decomposition of $G, f$ is a function of $a_{t}$ alone. In other words, there is a function $F$ on $\mathbb{R}_{+}$such that $f(x)=f\left(a_{t}\right)=F(C(t))$ if $x=k a_{t} K \in X$, where $C(t)=|t|, \cosh t$, or $\cos t$ for $X=\mathbb{R}^{n}, H^{n}$, or $S^{n}$ respectively. 
With a slight abuse of notation we write $f(t)$ for $F(C(t))$ whenever $f$ is a $K$ invariant function on $X$. Since $\tilde{\sigma}_{t}$ is a bi- $K$-invariant measure on $G$, it is easy to see that $\mathcal{A}_{t} f$ is bi- $K$-invariant whenever $f$ is bi- $K$-invariant on $G$.

Proposition 2.1. Let $f$ be a radial continuous function on $X$. Then $\mathcal{A}_{t} f(x)$ is given by the following formula, where $r=d(x, o)$ :

$$
\mathcal{A}_{t} f(r)=\frac{\Gamma(n / 2)}{\Gamma(1 / 2) \Gamma((n-1) / 2)}(\varphi(r) \varphi(t))^{2-n} \int_{|r-t|}^{r+t} f(u) \Delta^{n-3}(r, t, u) \varphi(u) d u .
$$

Here $\Delta$ is given by

$$
\Delta(r, t, u)=2 \sqrt{\varphi\left(\frac{r+t+u}{2}\right) \varphi\left(\frac{r+t-u}{2}\right) \varphi\left(\frac{r-t+u}{2}\right) \varphi\left(\frac{-r+t+u}{2}\right)},
$$

as above.

Proof. The proof of the formula depends on the cosine formula for the corresponding constant curvature geometry. First define $|w|=d(w, o)$ for $w \in X$, and $|\mathbf{w}|=$ $d(\mathbf{w} K, K)$ for $\mathbf{w} \in G$ under the identification $X=G / K$.

If $x$ and $y$ are two points in Euclidean space, then the distance between $x$ and $y$ is given by the formula

$$
|x-y|^{2}=|x|^{2}+|y|^{2}-2|x||y| \cos \theta,
$$

where $\theta$ is the angle between the vectors $x$ and $y$.

Similarly, the distances between the two points $\mathbf{x} K$ and $\mathbf{y} K$ on the sphere or hyperbolic space are given by the respective cosine formulas. If $r=|\mathbf{x}|$ and $t=|\mathbf{y}|$, then

$$
\cos d(\mathbf{x} K, \mathbf{y} K)=\cos \left|\mathbf{y}^{-1} \mathbf{x}\right|=\cos r \cos t+\cos \theta \sin r \sin t
$$

for the spherical geometry, and

$$
\cosh d(\mathbf{x} K, \mathbf{y} K)=\cosh \left|\mathbf{y}^{-1} \mathbf{x}\right|=\cosh r \cosh t-\cos \theta \sinh r \sinh t
$$

for the hyperbolic geometry.

Given two points $\mathbf{x} K$ and $\mathbf{y} K$ ), whose distances from the origin are given by $r=|\mathbf{x} K|$ and $t=\mid \mathbf{y} K) \mid$, the distance $d(\mathbf{x} K, \mathbf{y} K)$ depends only on the angle $\theta \leq \pi$ between the geodesics from $o$ to $\mathbf{x} K$ and $\mathbf{y} K$. We write $u=u(\theta)=\left|\mathbf{y}^{-1} \mathbf{x}\right|$ and differentiate the foregoing expression for the distance with respect to $\theta$. We obtain $\varphi(u) \frac{d u}{d \theta}=\sin \theta \varphi(r) \varphi(t)$. We now use the following fact:

$$
\varphi(r) \varphi(t) \sin \theta=\Delta(r, t, u) .
$$

This equality is clear in the Euclidean case, since $\Delta$ is twice the area of a triangle with side lengths $r, t, s$, and $r t \sin \theta$ is twice the area of a triangle with two sides of lengths $r$ and $t$ and interior angle $\theta$. For hyperbolic space, this fact is most conveniently established using vector calculus in the model for the hyperbolic plane given by the determinant-one surface in the Lie algebra of $S L_{2}(\mathbb{R})$. It follows, e.g., from the arguments in [Iv, pp. 89, 91, 107]. A similar proof can be given for spherical geometry. We conclude that

$$
\frac{d u}{d \theta}=\frac{\varphi(r) \varphi(t) \sin \theta}{\varphi(u)}=\frac{\Delta(r, t, u)}{\varphi(u)} .
$$


Now

$$
\mathcal{A}_{t} f(\mathbf{x})=\int_{S_{t}(o)} f\left(\mathbf{y}^{-1} \mathbf{x}\right) d \sigma_{t}(\mathbf{y})
$$

which is an integral over an $(n-1)$-dimensional sphere $S_{t}(o)$ in $X$. By assumption $f$ is $K$ bi-invariant, and $K$ is transitive on $S_{t}(o)$. It follows that $f$ is constant on the subset of $\mathbf{y} K \in S_{t}(o)$, where the value of $\left|\mathbf{y}^{-1} \mathbf{x}\right|=d(\mathbf{x} K, \mathbf{y} K)$ is a given constant $u(\theta)$ (where $\theta$ ranges over $0 \leq \theta \leq \pi$ ). The set in question is an $(n-2)$-dimensional sphere lying on $S_{t}(o)$, corresponding to the angle $\theta$. Now write the measure $d \sigma_{t}^{n}$ as $d \sigma_{u}^{n-1}(\omega) \sin \theta^{n-2} d \theta$, where $\sigma_{u}^{n-1}$ is is the normalized measure on an $(n-2)$-sphere of radius $u=u(\theta)$. Then

$$
\mathcal{A}_{t} f(\mathbf{x})=\frac{\omega_{n-2}}{\omega_{n-1}} \int_{0}^{\pi} f\left(\left|\mathbf{y}^{-1} \mathbf{x}(\theta)\right|\right) \sin ^{n-2} \theta d \theta=\frac{\omega_{n-2}}{\omega_{n-1}} \int_{0}^{\pi} f(u(\theta)) \sin ^{n-2} \theta d \theta,
$$

where $\omega_{n-1}=\frac{2 \pi^{n / 2}}{\Gamma(n / 2)}$. Now use the change of variable $u=\left|\mathbf{y}^{-1} \mathbf{x}\right|$, the Jacobian of which is given by (2.2). Finally, to determine the limits in the integral, note that from the cosine formula, it is easy to see that $|r-t| \leq u<r+t$ whenever $0 \leq \theta<\pi$. This completes the proof of Proposition 2.1.

As an immediate consequence, we get the following estimates for $\Delta$.

Proposition 2.2. Let $\Delta$ be as before. Then

$$
\begin{aligned}
\Delta(r, t, u) & \leq \varphi(r) \varphi(t), \\
\frac{\Delta(r, t, u)}{\varphi(u)} & \leq \varphi(r) \wedge \varphi(t),
\end{aligned}
$$

where $\wedge$ denotes the minimum.

Proof. As noted in the proof of Proposition 2.1, $\Delta=\varphi(r) \varphi(t) \sin \theta$, and since $0 \leq \theta \leq \pi$, the first estimate follows.

Now observe that $\Delta(r, t, u)$ is symmetric in $r, t$ and $u$. This follows from the expression for $\Delta$ in Proposition 2.1. Consequently, if $\Delta(r, t, u) \leq \varphi(r) \varphi(t)$, by symmetry we also have

$$
\begin{aligned}
\Delta(r, t, u) & \leq \varphi(u) \varphi(t) \\
\Delta(r, t, u) & \leq \varphi(u) \varphi(r) .
\end{aligned}
$$

The second inequality clearly follows from these two relations.

\section{The LoRentz SPACE $L^{p, q}(X)$}

In this section, we recall the definition of Lorentz space and discuss some auxiliary results that are needed in the proof of the main theorem. Let $(X, \mathcal{B}, \mu)$ be a sigma-finite measure space. Given $1 \leq p<\infty, 1 \leq q<\infty$, we say that a function $f$ belongs to $L^{p, q}(X, d \mu)$ if

$$
\|f\|_{p, q}=\left(\frac{q}{p} \int_{0}^{\infty}\left(t^{\frac{1}{p}} f^{*}(t)\right)^{q} \frac{d t}{t}\right)^{1 / q}<\infty
$$

Given $1 \leq p \leq \infty$, we say that $f \in L^{p, \infty}$ if

$$
\|f\|_{p, \infty}=\sup _{t>0} t^{\frac{1}{p}} f^{*}(t)=\sup _{\alpha>0} \alpha \lambda_{f}(\alpha)^{1 / p}<\infty .
$$


Here $f^{*}$ denotes the nonincreasing rearrangement of $f$; namely,

$$
f^{*}(t)=\inf \left\{s: \lambda_{f}(s)<t\right\},
$$

where

$$
\lambda_{f}(\alpha)=\mu\{x \in X:|f(x)|>\alpha\}
$$

denotes the distribution function of $f$.

We recall that $\|.\|_{p, q}$ is not a norm, since it fails to satisfy the triangle inequality, but $\|\cdot\|_{p, q}$ is equivalent to a norm satisfying the triangle inequality (see [SWe], Theorem 3.21, page 204). Besides this remark, we need two facts from Lorentz space theory. The first one concerns the norm of the characteristic function; namely, $\left\|\chi_{E}\right\|_{p, q}=\mu(E)^{\frac{1}{p}}$, whenever $E$ is a measurable subset of $X$ with finite measure. The second one is Hölder's inequality for Lorentz spaces:

$$
\left|\int_{X} f g d \mu\right| \leq\|f\|_{p, q, \mu}\|g\|_{p^{\prime}, q^{\prime}, \mu}
$$

whenever $f \in L^{p, q}(X, d \mu)$ and $g \in L^{p^{\prime}, q^{\prime}}(X, d \mu), \frac{1}{p}+\frac{1}{p^{\prime}}=1=\frac{1}{q}+\frac{1}{q^{\prime}}$ (see [BS]).

Since the group $G$ has a decomposition of the form $G=K A_{+} K$, the symmetric space has associated geodesic polar coordinates $K A_{+}$. Consequently, the $G$ invariant measure on $X$ decomposes as $d \mu=\varphi^{n-1}(t) d t d k$. Moreover, since we are dealing with $K$-invariant functions on $X$, the Lorentz space that we are concerned with is equivalent to the weighted Lorentz space on $\mathbb{R}_{+}$, namely

$$
L^{p, q}\left(\mathbb{R}_{+}, \varphi^{n-1}(t) d t\right),
$$

with weight $\varphi(t)=\sin t \chi_{[0, \pi]}, t$ or $\sinh t$ depending on the symmetric space. Let $\mu=\mu_{n}$ be the measure on $(0, \infty)$ given by $\mu_{n}(E)=\int_{E} \varphi^{n-1}(u) d u$.

Before formulating the proposition which is the key step in the proof of the main theorem, we define an auxiliary maximal operator, as follows:

$$
M f(r)=\sup _{0<t<r} \frac{\left\|f \chi_{(|r-t|, r+t)}\right\|_{n^{\prime}, 1 ; \mu_{n}}}{\left\|\chi_{(|r-t|, r+t)}\right\|_{n^{\prime}, 1 ; \mu_{n}}} .
$$

Proposition 3.1. Let $f$ be a continuous function with compact support on $\mathbb{R}_{+}$. Then

$$
\mathcal{M} f(r) \leq C_{n}\left[M f(r)+\|f\|_{n^{\prime}, 1 ; \mu_{n}} h(r)\right] .
$$

The function is given by

$$
h(r)=\frac{1}{\varphi^{(n-1)}(r)}
$$

in all cases, except hyperbolic space of dimension $n \geq 3$, where

$$
h(r)=\left[\frac{1}{\varphi^{n-1}(r)}+\frac{1}{\varphi^{\frac{(n-1)^{2}}{n}}(r)}\right] .
$$

The proof of Proposition 3.1 is particularly transparent in the case $n=2$ of the plane geometries. The volume estimates that it requires in the higher-dimensional case are somewhat more involved. We devote the next section to a proof of Proposition 3.1 in the 2-dimensional case, together with a proof of the main theorem based on Proposition 3.1.

Note. Henceforth we will always assume, in the case of radial averages on the sphere, that $0 \leq r, t \leq \frac{\pi}{4}$. This assumption is justified at the end of $\S 5$. 
Constant convention. Constants below will usually be denoted by $C$ or $C_{n}$, and depend only on the dimension of the space. Their value may change from line to line.

\section{The CASE $n=2$, AND PROOF OF THE MAIN THEOREM}

When $n=2$, the formula (2.1) for the spherical mean value operator becomes

$$
\mathcal{A}_{t} f(r)=\frac{1}{\pi} \int_{|r-t|}^{r+t} f(u) \frac{\chi_{(|r-t|, r+t)}}{\Delta(r, t, u)} \varphi(u) d u .
$$

The basic estimate needed in this case is given by

Lemma 4.1. Let $\Delta(r, t, u)$ be as in Proposition 2.1. Then

$$
\left\|\frac{\chi_{(|r-t|, r+t)}}{\Delta}\right\|_{2, \infty ; \mu_{2}} \leq 2\left[\mu_{2}(|r-t|, r+t)\right]^{-\frac{1}{2}}
$$

Proof. Without loss of generality, we assume $0<t \leq r$. We write

$$
\Delta(r, t, u)=\sqrt{[\psi(r+t)-\psi(u)][\psi(u)-\psi(r-t)]},
$$

where $\psi(u)=u^{2} / 2$, $\cosh u$ or $\cos u$ as the case may be. We compute the distribution function $\lambda$ of $\chi_{(r-t, r+t)} / \Delta$.

For each $\alpha>0$, let

$$
E(\alpha)=\left\{u \in(r-t, r+t): \frac{1}{\Delta}>\alpha\right\} .
$$

Then $E(\alpha)$ is given by

$$
E(\alpha)=\left\{\begin{array}{l}
(r-t, r+t) \text { for } \alpha<\frac{1}{B-A}, \\
\left\{u \in(r-t, r+t): \frac{1}{\sqrt{[B-S][S-A]}}>\alpha\right\} \text { for } \alpha \geq \frac{1}{B-A},
\end{array}\right.
$$

where $A=\psi(r-t), B=\psi(r+t)$ and $S=\psi(u)$. Now observe that $\mu_{2}(x, y)=$ $|(\psi(x), \psi(y))|$, the Euclidean measure of the interval $(\psi(x), \psi(y))$. Consequently,

$$
\mu_{2}(E(\alpha))=B-A \text { for } \alpha \leq \frac{1}{B-A} .
$$

For $\alpha>\frac{1}{B-A}$ we split the interval $(A, B)$ into $\left(A, \frac{A+B}{2}\right)$ and $\left(\frac{A+B}{2}, B\right)$. In the first interval $B-S \geq \frac{B-A}{2}$, and in the second interval $S-A \geq \frac{B-A}{2}$. Using these estimates and the foregoing observation regarding $\mu_{2}$, it is easy to see that $\mu_{2}(E(\alpha)) \leq \frac{4}{\alpha^{2}(B-A)}$ for $\alpha>\frac{1}{B-A}$. Consequently,

$$
\lambda(\alpha)\left\{\begin{array}{l}
=B-A \text { for } \alpha \leq \frac{1}{B-A}, \\
\leq \frac{4}{\alpha^{2}(B-A)} \text { for } \alpha>\frac{1}{B-A} .
\end{array}\right.
$$

It follows that

$$
\sup _{\alpha>0} \alpha(\lambda(\alpha))^{\frac{1}{2}} \leq \frac{2}{\sqrt{B-A}}=\frac{2}{\mu_{2}(r-t, r+t)^{1 / 2}} .
$$

That is,

$$
\left\|\frac{\chi_{(r-t, r+t)}}{\Delta}\right\|_{2, \infty ; \mu_{2}} \leq \frac{2}{\left\|\chi_{(r-t, r+t)}\right\|_{2, \infty ; \mu_{2}}}=\frac{2}{\mu_{2}(r-t, r+t)^{1 / 2}} .
$$


Proof of Proposition 3.1, for $n=2$. Clearly

$$
\sup _{t>0} \mathcal{A}_{t} f(r) \leq \sup _{t<r} \mathcal{A}_{t} f(r)+\sup _{t>r} \mathcal{A}_{t} f(r)
$$

We estimate each of these terms separately. Using the formula of Proposition 2.1 for $\mathcal{A}_{t} f(r)$ and Hölder's inequality, we obtain

$$
\left|\mathcal{A}_{t} f(r)\right| \leq \frac{1}{\pi}\left\|\frac{\chi_{(|r-t|, r+t)}}{\Delta(r, t, \cdot)}\right\|_{2, \infty ; \mu_{2}}\left\|f \chi_{(r-t, r+t)}\right\|_{2,1 ; \mu_{2}} .
$$

When $t<r$, in view of Lemma 4.1, we see that the above is less than or equal to

$$
\frac{2}{\pi} \frac{\left\|f \chi_{(r-t, r+t)}\right\|_{2,1 ; \mu_{2}}}{\left\|\chi_{(r-t, r+t)}\right\|_{2, \infty ; \mu_{2}}} .
$$

Since $\left\|\chi_{(r-t, r+t)}\right\|_{2, \infty ; \mu_{2}}=\left\|\chi_{(r-t, r+t)}\right\|_{2,1 ; \mu_{2}}$ (see $\left.\S 3\right)$, it follows that (by definition of $M)$

$$
\sup _{t<r}\left|\mathcal{A}_{t} f(r)\right| \leq \frac{2}{\pi} M f(r) .
$$

To deal with the case $t>r$, note first that

$$
\int_{t-r}^{t+r} \varphi(u) d u=\mu_{2}((t-r, t+r))=2 \varphi(r) \varphi(t) .
$$

Using Hölder's inequality as before, together with Lemma 4.1 and the foregoing fact, we get

$$
\mathcal{A}_{t} f(r) \leq \frac{2}{\pi} \frac{\|f\|_{2,1 ; \mu_{2}}}{(2 \varphi(r) \varphi(t))^{1 / 2}} .
$$

Using the fact that $\varphi(u)$ is increasing (recall that in the sphere case we assume $0 \leq r, t, \leq \pi / 4)$, we conclude that

$$
\sup _{t>r}\left|\mathcal{A}_{t} f(r)\right| \leq \frac{\sqrt{2}}{\pi} \frac{\|f\|_{2,1 ; \mu_{2}}}{\varphi(r)} .
$$

This completes the proof of Proposition 3.1 for the case $n=2$.

We proceed to prove the main theorem, assuming Proposition 3.1 (in all dimensions).

Proof Theorem 1.1. A simple computation shows that the function $\frac{\chi_{(0, \infty)}}{\varphi(r)^{n-1}}$ is in the space $L^{n^{\prime}, \infty}\left(\mathbb{R}_{+}, d \mu_{n}\right), n \geq 2$, whenever $\varphi$ has polynomial growth, and also for $\varphi$ of exponential growth when $n=2$. When $n \geq 3$ and $\varphi$ has exponential growth, it can be shown similarly that $\frac{\chi(0, \infty)}{\varphi(r)^{p}} \in L^{n^{\prime}, \infty}\left(\mathbb{R}_{+}, d \mu_{n}\right)$ if and only if $\frac{(n-1)^{2}}{n} \leq p \leq n-1$. Consequently $h(r) \in L^{n^{\prime}, \infty}\left(\mathbb{R}_{+}, d \mu_{n}\right)$. So it is enough to show that the operator $M$ satisfies the inequality

$$
\|M f\|_{n^{\prime}, \infty ; \mu_{n}} \leq C_{n}\|f\|_{n^{\prime}, 1 ; \mu_{n}} .
$$

Since $\|\cdot\|_{n^{\prime}, 1 ; \mu_{n}}$ is monotone, and since every nonnegative measurable function can be approximated by an increasing sequence of finite linear combinations of characteristic functions of sets of finite measure, it is enough to prove the theorem for functions of the form $f=\chi_{E}$ for any set $E \subset \mathbb{R}_{+}$of finite $\mu_{n}$-measure (see SWe, Thm. 3.13, p. 195). 
Now

Thus it follows that

$$
M \chi_{E}(r)=\sup _{0<t<r}\left[\frac{\mu_{n}(E \cap(r-t, r+t))}{\mu_{n}(r-t, r+t)}\right]^{1 / n^{\prime}} .
$$

$$
\left\{r>0: M \chi_{E}(r)>\alpha\right\}=\left\{r>0: \sup _{0<t<r} M_{n} \chi_{E}(r)>\alpha^{n^{\prime}}\right\},
$$

where

$$
M_{n} f(r)=\sup _{0<t<r} \frac{1}{\mu_{n}(r-t, r+t)} \int_{r-t}^{r+t} f(u) d \mu_{n}(u)
$$

is the Hardy-Littlewood maximal operator on $\mathbb{R}_{+}$with measure

$$
d \mu_{n}(u)=\varphi^{n-1}(u) d u
$$

It is well known that $\mu_{n}$ is of weak type $(1,1)$. A proof of this fact for measures $\mu_{n}$ satisfying the doubling condition, and hence for measures of polynomial growth, can be seen, e.g., in [T], page 225. Of course, in the Euclidean case, it is also a consequence of Wiener's maximal inequality for ball averages on $\mathbb{R}^{n}$, when applied to radial functions. Similarly, for the measures $\mu_{n}$ of exponential growth, the desired inequality follows from Stromberg's weak-type $(1,1)$ maximal inequality for ball averages on hyperbolic spaces (see $[\mathrm{S}]$ ), applied to radial functions.

Thus we have

$$
\mu_{n}\left\{r>0: M\left(\chi_{E}\right)(r)>\alpha\right\} \leq \frac{C}{\alpha^{n^{\prime}}} \mu_{n}(E)
$$

Consequently,

$$
\sup _{\alpha>0} \alpha\left(\mu_{n}\left\{r>0: M \chi_{E}(r)>\alpha\right\}\right)^{\frac{1}{n^{\prime}}}=\left\|M \chi_{E}\right\|_{n^{\prime}, \infty ; \mu_{n}} \leq C \mu_{n}(E)^{1 / n^{\prime}} .
$$

This completes the proof of the main theorem, since $\mu_{n}(E)^{\frac{1}{n^{\prime}}}=\left\|\chi_{E}\right\|_{n^{\prime}, 1, \mu_{n}}$.

\section{THE CASE $n \geq 3$}

To prove Proposition 3.1 in the case $n \geq 3$, we need the following volume estimates. Let $\eta(t)$ stand for the function $\frac{\varphi(t)}{\varphi^{\prime}(t)}$.

Lemma 5.1. Let $0<t \leq r$.

$$
\begin{aligned}
& \text { (i) }\left\|\frac{\chi_{(r-t, r+t)}}{\varphi^{n-2}}\right\|_{n, \infty ; \mu_{n}} \leq 2^{n-2} \frac{\mu(r-t, r+t)^{1 / n}}{\varphi^{(n-2)}(r+t)} \text { if } \eta(t) \leq \frac{1}{3} \eta(r) . \\
& \text { (ii) }\left\|\frac{\chi_{(r-t, r+t)}}{\varphi}\right\|_{n, \infty ; \mu_{n}} \leq\left\{\begin{array}{l}
C \text { if } \varphi \text { has polynomial growth } \\
\frac{C}{[1+\varphi(r-t)]^{1 / n}} \text { if } \varphi \text { has exponential growth. }
\end{array}\right.
\end{aligned}
$$

We postpone the proof of Lemma 5.1 to the end of this section, and show first how to prove Proposition 3.1 for dimensions $n \geq 3$ from it.

Proof of Proposition 3.1, $n \geq 3$. We have

$$
\sup _{t>0} \mathcal{A}_{t} f(r) \leq \sup _{\eta(t) \leq \frac{1}{3} \eta(r)} \mathcal{A}_{t} f(r)+\sup _{\eta(t) \leq \frac{1}{3} \eta(r)} \mathcal{A}_{t} f(r) .
$$

First let us consider the case $\eta(t) \leq \frac{1}{3} \eta(r)$, so that in particular $t \leq r$. Rewriting (2.1) as

$$
\mathcal{A}_{t} f(r)=C_{n}[\varphi(t) \varphi(r)]^{2-n} \int_{r-t}^{r+t} f(u) \chi_{(r-t, r+t)} \frac{\Delta^{n-3}(r, t, u)}{\varphi^{n-2}(u)} \varphi^{n-1}(u) d u
$$


and using Hölder's inequality for Lorentz spaces, we get

$$
\begin{aligned}
\left|\mathcal{A}_{t} f(r)\right| & \leq C_{n}[\varphi(r) \varphi(t)]^{2-n}\left\|\frac{\chi_{(r-t, r+t)}}{\varphi^{n-2}} \Delta^{n-3}(r, t, \cdot)\right\|\left\|_{n, \infty ; \mu_{n}}\right\| f \chi_{(r-t, r+t)} \|_{n^{\prime}, 1 ; \mu_{n}} \\
& \leq C_{n} C(r, t) M f(r)
\end{aligned}
$$

where

$$
C(r, t)=[\varphi(r) \varphi(t)]^{2-n}\left\|\frac{\chi_{(r-t, r+t)}}{\varphi^{n-2}} \Delta^{n-3}(r, t, \cdot)\right\|_{n, \infty ; \mu_{n}}\left\|\chi_{(r-t, r+t)}\right\|_{n^{\prime}, 1 ; \mu_{n}} .
$$

It now suffices to prove that $C(r, t)$ is uniformly bounded in $r$ and $t$ whenever $\eta(t) \leq \frac{1}{3} \eta(r)$, in order to obtain the desired conclusion, namely

$$
\sup _{\eta(t) \leq \frac{1}{3} \eta(r)} \mathcal{A}_{t} f(r) \leq C_{n} M f(r) .
$$

To prove uniform boundedness of $C(r, t)$, we use the first estimate for $\Delta$ in Proposition 2.2 and (i) of Lemma 5.1, and obtain

$$
C(r, t) \leq \frac{1}{\varphi(r) \varphi(t)} \frac{\mu(r-t, r+t)^{1 / n} \mu(r-t, r+t)^{1 / n^{\prime}}}{\varphi^{n-2}(r+t)} .
$$

Since $n^{\prime}$ is the index conjugate to $n$, using $\varphi(u) \leq \varphi(r+t)$ for $u \leq r+t$ (recall the restriction on $r, t$ in the sphere case) we get

$$
\begin{aligned}
C(r, t) & \leq \frac{\mu_{n}(r-t, r+t)}{\varphi(r) \varphi(t) \varphi^{n-2}(r+t)} \\
& \leq \frac{1}{\varphi(r) \varphi(t)} \int_{r-t}^{r+t} \varphi(u) d u .
\end{aligned}
$$

The boundedness of $C(r, t)$ follows from the observation that

$$
\int_{r-t}^{r+t} \varphi(u) d u=2 \varphi(r) \varphi(t) .
$$

Now let us consider the case $\eta(t) \geq \frac{1}{3} \eta(r)$. Again, using Hölder's inequality,

$$
\left|\mathcal{A}_{t} f(r)\right| \leq C_{n}[\varphi(r) \varphi(t)]^{2-n}\|f\|_{n^{\prime}, 1 ; \mu_{n}}\left\|\frac{\Delta^{n-3}(r, t, \cdot) \chi_{(|r-t|, r+t)}}{\varphi^{n-2}}\right\|_{n, \infty ; \mu_{n}} .
$$

We first estimate

$$
[\varphi(r) \varphi(t)]^{2-n}\left\|\frac{\Delta^{n-3}(r, t, \cdot)}{\varphi^{n-3}} \frac{\chi_{(|r-t|, r+t)}}{\varphi}\right\|_{n, \infty ; \mu_{n}} .
$$

Using the estimate in (ii) of Proposition 2.2, namely $\frac{\Delta(r, t, u)}{\varphi(u)} \leq \varphi(t)$, we get

$$
\begin{gathered}
{[\varphi(r) \varphi(t)]^{2-n}\left\|\frac{\Delta^{n-3}(r, t, \cdot) \chi_{(|r-t|, r+t)}}{\varphi^{n-3} \varphi}\right\|_{n, \infty ; \mu_{n}}} \\
\leq \frac{1}{\varphi^{n-2}(r) \varphi(t)}\left\|\frac{\chi_{(|r-t|, r+t)}}{\varphi}\right\|_{n, \infty ; \mu_{n}} \\
\quad \leq \frac{1}{\varphi^{n-2}(r)} \frac{3}{\varphi^{\prime}(t) \eta(r)}\left\|\frac{\chi_{(|r-t|, r+t)}}{\varphi}\right\|_{n, \infty ; \mu_{n}} .
\end{gathered}
$$


The last step follows by writing $\varphi(t)=\varphi^{\prime}(t) \eta(t)$ and using the inequality $\eta(t) \geq$ $\frac{1}{3} \eta(r)$. Now, writing $\eta(r)$ as $\frac{\varphi(r)}{\varphi^{\prime}(r)}$, we see that the RHS of the above equation becomes

$$
\frac{3}{\varphi^{n-1}(r)} \frac{\varphi^{\prime}(r)}{\varphi^{\prime}(t)}\left\|\frac{\chi_{(|r-t|, r+t)}}{\varphi}\right\|_{n, \infty ; \mu_{n}} .
$$

It follows from (ii) of Lemma 5.1 that $\left\|\frac{\chi(|r-t|, r+t)}{\varphi}\right\|_{n, \infty ; \mu_{n}}$ is bounded uniformly in $r$ and $t$. Now observe that $\frac{\varphi^{\prime}(r)}{\varphi^{\prime}(t)}$ is bounded whenever $\varphi(u)=u$, or $\sin u$ (again since we are assuming in the sphere case that $0<r, t \leq \pi / 4)$. The expression is also bounded for $\sinh u$ whenever $r-t \leq 1$. Therefore, in these cases,

$$
\sup _{\eta(t)>\frac{1}{3} \eta(r)} \mathcal{A}_{t} f(r) \leq C \frac{\|f\|_{n^{\prime}, 1 ; \mu_{n}}}{\varphi^{n-1}(r)} .
$$

Now it remains to settle the case $r-t>1$ for $\varphi(u)=\sinh u$. In this case, we use the estimate

of Lemma 5.1.

$$
\left\|\frac{\chi_{(|r-t|, r+t)}}{\varphi}\right\|_{n, \infty ; \mu_{n}} \leq \frac{1}{[1+\varphi(r-t)]^{1 / n}}
$$

Using the fact that $\varphi^{\prime}(u) \approx e^{u}$ and $\varphi(u) \approx e^{u}$ for $u>1$, we see that

$$
\begin{aligned}
\frac{1}{\varphi^{n-2}(r)} \frac{3}{\varphi^{\prime}(t) \eta(r)}\left\|\frac{\chi_{(|r-t|, r+t)}}{\varphi}\right\|_{n, \infty ; \mu_{n}} & \leq \frac{1}{\varphi^{n-2}(r)} \frac{3}{\varphi^{\prime}(t) \eta(r)} \frac{1}{\varphi^{1 / n}(r-t)} \\
& \approx \frac{1}{\varphi^{n-1}(r)} \frac{e^{r-t}}{e^{(r-t) / n}} \\
& \approx \frac{[\varphi(r-t)]^{\frac{n-1}{n}}}{\varphi^{n-1}(r)} \\
& \leq \frac{\varphi(r)^{(n-1) / n}}{\varphi^{n-1}(r)}=\frac{1}{\varphi(r)^{\frac{(n-1)^{2}}{n}} .}
\end{aligned}
$$

This completes the proof of Proposition 3.1 for the case $n \geq 3$.

Proof of Lemma 5.1. Consider the Lorentz norm of $\left\|\frac{\chi(r-t, r+t)}{\varphi^{n-2}(u)}\right\|_{n, \infty ; \mu_{n}}$, given by $\sup _{\alpha>0} \alpha(\lambda(\alpha))^{1 / n}$, where $\lambda$ is now the distribution function for $\frac{\chi_{(r-t, r+t)}}{\varphi^{n-2}}$. An easy computation shows that

$$
\lambda(\alpha)=\left\{\begin{array}{l}
\mu_{n}(r-t, r+t) \text { if } \alpha<\frac{1}{\varphi^{n-2}(r+t)}, \\
\mu_{n}\left(r-t, \varphi^{-1}\left(\alpha^{\frac{-1}{n-2}}\right)\right) \text { if } \frac{1}{\varphi^{n-2}(r+t)}<\alpha<\frac{1}{\varphi^{n-2}(r-t)} .
\end{array}\right.
$$

Therefore,

$$
\begin{aligned}
& \sup _{\alpha>0} \alpha(\lambda(\alpha))^{\frac{1}{n}} \\
& =\max \left\{\frac{\mu_{n}(r-t, r+t)^{1 / n}}{\varphi^{n-2}(r+t)}, \frac{1}{\frac{1}{\varphi^{n-2}(r+t)}<\alpha<\frac{1}{\varphi^{n-2}(r-t)}} \alpha\left[\mu_{n}\left(r-t, \varphi^{-1}\left(\alpha^{\frac{-1}{n-2}}\right)\right)\right]^{1 / n}\right\} .
\end{aligned}
$$

Now, observing that $\eta(t) \leq \frac{1}{3} \eta(r)$ is equivalent to $\varphi(r+t) \leq 2 \varphi(r-t)$, we take the obvious estimate of both $\alpha$ and the size of the interval, and conclude that

$$
\alpha\left[\mu\left(r-t, \varphi^{-1}\left(\alpha^{\frac{-1}{n-2}}\right)\right)\right]^{1 / n} \leq 2^{n-2} \frac{\mu_{n}(r-t, r+t)^{1 / n}}{\varphi^{n-2}(r+t)}
$$


whenever $\frac{1}{\varphi^{n-2}(r+t)}<\alpha<\frac{1}{\varphi^{n-2}(r-t)}$. This proves (i).

To prove (ii), first we consider the case when $\varphi$ has polynomial growth. This is the case with the sphere as well as the Euclidean space. As before, we have

$$
\begin{aligned}
& \left\|\frac{\chi_{r-t, r+t}}{\varphi(u)}\right\|_{n, \infty, \mu} \\
& \quad=\max \left\{\frac{\mu_{n}(r-t, r+t)^{1 / n}}{\varphi(r+t)}, \frac{1}{\frac{1}{\varphi(r+t)}<\alpha<\frac{1}{\varphi(r-t)}} \alpha\left[\mu_{n}\left(r-t, \varphi^{-1}\left(\frac{1}{\alpha}\right)\right)\right]^{1 / n}\right\} .
\end{aligned}
$$

We estimate each of these terms separately. Clearly,

$$
\begin{aligned}
\frac{\mu_{n}(r-t, r+t)}{\varphi^{n}(r+t)} & =\frac{\int_{r-t}^{r+t} \varphi^{n-1}(u) d u}{\varphi^{n}(r+t)} \\
& \leq \frac{\int_{r-t}^{r+t} u^{n-1} d u}{\varphi^{n}(r+t)}
\end{aligned}
$$

The above expression behaves like

$$
\frac{r^{n-1} t}{\varphi^{n}(r+t)}=\frac{t}{r}\left[\frac{r}{\varphi(r+t)}\right]^{n}
$$

which is uniformly bounded since $t<r$ and since $\varphi(r+t)>C(r+t)$ (recall that for the sphere we are assuming that both $r$ and $t$ are less than $\pi / 4$ ). Similarly,

$$
\alpha^{n}\left[\mu_{n}\left(r-t, \varphi^{-1}\left(\frac{1}{\alpha}\right)\right)\right] \leq C_{n} \alpha^{n} \int_{r-t}^{\varphi^{-1}\left(\frac{1}{\alpha}\right)} u^{n-1} d u .
$$

The above expression is also bounded, since $\varphi^{-1}\left(\frac{1}{\alpha}\right)<\frac{c}{\alpha}$ for some constant $c$.

Now let us consider the case when $\varphi$ has exponential growth. This is the case with hyperbolic space, where $\varphi(u)=\sinh u$. We assume $r+t>2$, since $\varphi(u)$ behaves like a polynomial for $0 \leq u \leq 2$; so the previous arguments apply. Then

$$
\begin{aligned}
\frac{\mu_{n}(r-t, r+t)}{\varphi^{n}(r+t)} & \leq \frac{\int_{r-t}^{r+t} e^{(n-1) u}}{\varphi^{n}(r+t)} \\
& \leq \frac{e^{(n-1)(r+t)}}{(n-1)\left(e^{r+t}-e^{-(r+t)}\right)^{n}} \\
& \leq \frac{e^{(n-1)(r+t)}}{(n-1)\left(\frac{1}{2} e^{r+t}\right)^{n}} \text { since }(r+t)>2 \\
& \leq \frac{C_{n}}{e^{r+t}} \leq \frac{C_{n}}{1+\varphi(r-t)} \text { since both } r, t>0 .
\end{aligned}
$$

Also, using a simple change of variable, we see that

$$
\alpha^{n}\left[\mu_{n}\left(r-t, \varphi^{-1}\left(\frac{1}{\alpha}\right)\right)\right]=C_{n} \alpha^{n} \int_{\varphi(r-t)}^{\frac{1}{\alpha}} \frac{s^{n-1}}{\sqrt{1+s^{2}}} d s .
$$

Using the inequality $\frac{s}{\sqrt{1+s^{2}}} \leq 1$, we get the estimate

$$
\alpha^{n}\left[\mu_{n}\left(r-t, \varphi^{-1}\left(\frac{1}{\alpha}\right)\right)\right] \leq \frac{\alpha-\alpha^{n} \varphi^{n-1}(r-t)}{n-1} .
$$


A derivative computation shows that this, as a function of $\alpha$, is maximum when $\alpha=\frac{n^{-\frac{1}{n-1}}}{\varphi(r-t)}$. Consequently,

$$
\alpha\left[\mu_{n}\left(r-t, \varphi^{-1}\left(\frac{1}{\alpha}\right)\right)\right]^{\frac{1}{n}} \leq \frac{n^{\frac{-1}{n(n-1)}}}{\varphi^{\frac{1}{n}}(r-t)} .
$$

Using the inequality $\sqrt{1+s^{2}}>1$ in (5.2), we also see that

$$
\alpha\left[\mu_{n}\left(r-t, \varphi^{-1}\left(\frac{1}{\alpha}\right)\right)\right]^{\frac{1}{n}} \leq C_{n} .
$$

It follows that

$$
\alpha\left[\mu_{n}\left(r-t, \varphi^{-1}\left(\frac{1}{\alpha}\right)\right)\right]^{\frac{1}{n}} \leq \frac{C_{n}}{[1+\varphi(r-t)]^{\frac{1}{n}}} .
$$

This proves (ii), and concludes the proof of Lemma 5.1.

Radial averages on the spheres. We now explain the reduction regarding the spherical mean value operator on the sphere. As noted in the argument above, it was useful to work in the range where the density function $\varphi(t)=\sin t$ is monotonic, namely $[0, \pi / 2]$. The symmetry of the sphere, as well as of the integral kernel of Proposition 2.1, allows us to reduce the analysis to this case, and show that the spherical maximal operator $\sup _{0<t<\pi}\left|\mathcal{A}_{t} f(r)\right|$ on the sphere is controlled by $\sup _{t>0}\left|\mathcal{A}_{t} f(r)\right|$ for $0 \leq r, t \leq \frac{\pi}{4}$, as follows.

First, if $f$ is a $K$-invariant function on the sphere, then, as we observed before, $f$ can be identified with a function on $[0, \pi]$. Letting $f(x)=F(\cos r)$, where $r=d(0, x)$ is the geodesic distance of $x$ from the north pole, and setting $\check{f}(x)=$ $F(\cos (\pi-r))$, we see that the function $g(x)=f(x)+\check{f}(x)$ is symmetric about the equator, i.e., $g(x)=\check{g}(x)$. Moreover, if $f$ is nonnegative, we have $f(x) \leq g(x)$. So when dealing with the spherical maximal operator we can assume, without loss of generality, that $f$ is nonnegative and symmetric about the equator.

Since $\Delta(r, t, u)=\Delta(\pi-r, t, \pi-u)$, an easy computation shows that $\mathcal{A}_{t} f(x)=$ $\left(\mathcal{A}_{t} f \check{f}(x)\right.$ whenever $\check{f}(x)=f(x)$. Consequently, if $f=\check{f}$, the function $\mathcal{A}_{t} f(r)$ is completely determined by its values on $0 \leq r \leq \frac{\pi}{2}, 0 \leq t \leq \pi$. Now note that we can assume, in addition, that $0 \leq t \leq \frac{\pi}{2}$. This follows from the relation $\mathcal{A}_{t} f(r)=\mathcal{A}_{r} f(t)$, which is a consequence of the symmetry of $\Delta$ in $r$ and $t$.

Now we go one step further. We claim that $\mathcal{A}_{t} f(r)$ is controlled by the values it assumes when both $r$ and $t$ are less than $\pi / 4$. This follows from the inequality

$$
\sup _{0<t<\pi / 2} \mathcal{A}_{t} f(r) \chi_{(0, \pi / 2)}(r) \leq C \sup _{0<t<\pi / 4} \mathcal{A}_{t} f_{2}\left(\frac{r}{2}\right) \chi_{(0, \pi / 2)}(r),
$$

where $f_{2}(u)=f(2 u)$. To establish this fact, note that, assuming $r, t \leq \pi / 2$, we have

$$
\sup _{0<t<\pi / 2} \mathcal{A}_{t} f(r)=\sup _{0<\tau<\pi / 4} \mathcal{A}_{2 \tau} f(r) .
$$

Writing $\rho=\frac{r}{2}$, we see that

$$
\mathcal{A}_{2 \tau} f(r) \chi_{(0, \pi / 2)}(r)=\mathcal{A}_{2 \tau} f(2 \rho) \chi_{(0, \pi / 4)}(\rho) .
$$

Now

$$
\mathcal{A}_{2 \tau} f(2 \rho)=(\sin (2 \rho) \sin (2 \tau))^{2-n} \int_{|2 \tau-2 \rho|}^{2 \tau+2 \rho} f(u) \Delta^{n-3}(2 \rho, 2 \tau, u) \sin u d u
$$


Using a change of variable and the fact that $\sin (2 v) \leq 2 \sin v$ for $0 \leq v \leq \frac{\pi}{2}$, we conclude that $\Delta(2 r, 2 t, 2 v) \leq 2^{4} \Delta(r, t, v)$. Since $\sin 2 t \sim 2 \sin t$ for $0<t<\pi / 4$, we see that

$$
\mathcal{A}_{2 \tau} f(2 \rho) \leq C \mathcal{A}_{\tau} f_{2}(\rho) \text { for } 0<\rho<\pi / 4 .
$$

This proves the inequality.

Finally, since the Lorentz spaces $L^{p, q}(X, d \mu)$ are defined in terms of distribution functions, they are dilation-invariant as long as the measure $\mu$ is of polynomial growth. Since this is the case with the sphere, the foregoing inequality allows us to assume that the function $\varphi(u)=\sin u$ is increasing in the region $(|r-t|, r+t)$.

Acknowledgement. This work was carried out while the second author was a postdoctoral fellow in the department of mathematics at Technion during the year 1998-1999. He wishes to thank the faculty and the other staff members for their warm hospitality during his stay at Technion.

\section{REFERENCES}

[B1] J. BOURGAIN, Averages in the plane over convex curves and maximal operators, $J$. d'Analyse Math. 47 (1986), 69 - 85. MR 88f:42036

[B2] J. BOURGAIN, Estimations de certaines fonctions maximales. C. R. Acad. Sci. Paris, Sér. I Math. 301, 499-502, 1985. MR 87b:42023

[BS] C. BENNETT and R. SHARPLEY, Interpolation of Operators, Pure and Applied Math., vol. 129, Academic Press, New York, 1988. MR 89e:46001

[C] M. COWLING, On Littlewood-Paley-Stein theory, Proceedings of the seminar in Harmonic Analysis (Pisa, 1980) Rend. Circ. Math. Palermo (2) (1981) suppl. 1, pp. 21-55. MR 83h:42024

[CN] M. COWLING and A. NEVO, Uniform estimates for spherical functions on complex semisimple Lie groups. Geometric and Functional Analysis 11 (2001), 900-932.

[Ch] I. CHAVEL, Eigenvalues in Riemannian Geometry, Academic Press, 1984. MR 86g:58140

[FJ-K] M. FLENSTED-JENSEN AND T. H. KOORNWINDER, The convolution structure for Jacobi function expansion, Arkiv für Matematik, vol. 11 (1973) pp. 245-262. MR 49:5688

[H1] S. HELGASON, Differential geometry, Lie groups and symmetric spaces, Academic Press, New York, 1978. MR 80k:53081

[H2] S. HELGASON, Geometric analysis on symmetric spaces, Amer. Math. Soc. Mathematical Surveys and Monographs Series, Vol. 39, 1994. MR 96h:43009

[Hi] N. J. HICKS, Notes on Differential Geometry, Van Nostrand, 1965. MR 31:3936

[I] A. D. IONESCU, Fourier integral operators on non-compact symmetric spaces of real rank one. J. Funct. Anal., vol. 174, pp. 274-300 (2000). MR 2001h:43009

[Iv] B. IVERSEN, Hyperbolic geometry, London Math. Soc. Student Texts, vol. 25, Cambridge Univ. Press, 1992. MR 94b:51023

[KR] R. KERMAN and P. K. RATNAKUMAR, Spherical means of radial functions, Preprint.

[K] T. H. KOORNWINDER, Jacobi functions and analysis on non-compact semisimple Lie groups. In : R. A. Askey et al. (eds.), Special Functions : Group Theoretical Aspects and Applications, pp. 1-85 , 1984, D. Reidel Publishing Company. MR 86m:33018

[L] M. LECKBAND, A note on the spherical maximal operator for radial functions, Proc. Amer. Math. Soc. vol. 100 (1987) pp. 635-640. MR 88i:42032

[N1] A. NEVO, Pointwise ergodic theorem for radial averages on simple Lie groups I, Duke Math J. 76 (1994), 113 - 140. MR 96c:28027

[N2] A. NEVO, Pointwise ergodic theorem for radial averages on simple Lie groups II, Duke Math J. 86 (1997), 239-259. MR 98m:28041

[NS] A. NEVO and E. M. STEIN, Analogs of Wiener's ergodic theorems for semisimple groups I, Ann. of Math. 145 (1997) 565-595. MR 98m:22007

[NT] A. NEVO and S. THANGAVELU, Pointwise ergodic theorems for radial averages on the Heisenberg group, Adv. in Math. vol. 127 (1997) pp. 307-334. MR 98f:22005

[S1] E. M. STEIN, Maximal functions: spherical means, Proc. Nat. Acad. Sci. U.S.A, 73 (1976), 2174 - 2175. MR 54:8133a 
[S2] E. M. STEIN, Harmonic Analysis : Real Variable Methods, Orthogonality and Oscillatory Integrals, Princeton University Press, Princeton, New Jersey, 1993. MR 95c: 42002

[SWa] E. M. STEIN and S. WAINGER, Problems in harmonic analysis related to curvature, Bull. Amer. Math. Soc. vol. 84, (1978) pp. 1239-1295. MR 80k:42023

[SWe] E. M. STEIN and G. WEISS, Introduction to Fourier Analysis on Euclidean Spaces, Princeton Univ. Press, Princeton, N.J. (1971). MR 46:4102

[S] J. O. STROMBERG, Weak type $L^{1}$ estimate for maximal functions on non compact symmetric spaces, Ann. of Math., vol. 114 (1981) 115 - 126. MR 82k:43010

[T] A. TORCHINSKY, Real Variable Methods in Harmonic Analysis, Academic Press, San Diego (1986). MR 88e:42001

[W] N. J. WILDBERGER, Hypergroups, symmetric spaces, and wrapping maps. In : Probability measures on groups and related structures, vol. XI, pp. 406-425, World Sci. Publishing, River Edge, NJ, 1995. MR 98b:43014

Institute of advanced studies in mathematics, Technion-Israel Institute of Technology, Haifa 32900, IsRael

E-mail address: anevo@tx.technion.ac.il

Institute of advanced studies in mathematics, Technion-Israel Institute of Technology, HAIFA 32900, ISRAEL

E-mail address: pkrsm@uohyd.ernet.in 